\title{
Evaluation of Serological Tests for Immunodiagnosis of Pulmonary Tuberculosis using A60 and Lipoarabinomannan Antigens
}

\author{
El Kabeer $M^{1}$, Seddek $M N^{1}$, El Tayeb $S^{2}$, EI Ridi $R^{3}$. \\ ${ }^{1}$ Faculty of Science Benha University, ${ }^{2}$ Clinical Pathology \\ Department- Faculty of Medicine Al Azhar University, ${ }^{4}$ Faculty of \\ Science Cairo University
}

\begin{abstract}
Background: Problems in diagnosis of tuberculosis using smear and culture for acid fast bacilli ( $A F B)$ techniques have necessitated the exploration of the utility of serodiagnosis to support the clinical suspicion of tuberculosis. Three serological tests, namely Anti-A60 IgG enzyme-linked immunosorbent assay (ELISA), Anti-A60IgM (ELISA) using A60 antigen and Mycodot test using lipoarabinomannan (LAM) antigen were evaluated as tools of diagnosing pulmonary tuberculosis against smear and culture methods. Materials and Methods: ELISA was used for the detection of IgG\& IgM using A60 antigen, while Mycodot test was performed utilizing Lipoarabinomannan (LAM) antigen bound to plastic combs in parallel with other familiar diagnostic methods in 50 patients with pulmonary tuberculosis (Group I),25 patients with chest diseases other than tuberculosis (Group II) and 25 apparently healthy individuals(Group III) Members of (Group II) \& (Group III) participate as control groups. All members of the three groups were examined for Ziehl-Neelsen (ZN) smear stains, culture for acid fast bacilli (AFB) and Tuberculin skin test. Results: ELISA IgG results were positive in 42 (84\%) tuberculous patients of Group I compared to 10 (40\%) non tuberculous patients in Group II and 8 (32\%) individuals in Group III. ELISA IgM results were positive in 30 (60\%) tuberculous patients of Group I compared to 3(12\%) non-tuberculous patients of Group II and 2 (8\%) individuals of Group III. Mycodot test results were positive in 33 (66\%) tuberculous patients in Group I compared to 6 (24\%) non-tuberculous patients in Group II and 3 (12\%) individuals of Group III. The overall sensitivities and specificities of the three tests (Mycodot, IgG and IgM) were obtained on basis of the receiver operating characteristic (ROC) curve for each test and comparison of (ROC) curves of the three tests and they were $(66,82 \%)$ for Mycodot test, $(80,92 \%)$ for Anti-A60 IgG and (82,74\%) for Anti-A60 IgM. Positive predictive values and negative predictive values were (78.57, 70.69\%) for Mycodot test, (90.91, 82.14\%) for IgG and (75.93, 80.43\%) for IgM. Results of Ziehl-Neelsen (ZN) smear stains and culture for Acid fast Bacilli (AFB) were positive in all members of group (I), while were negative in all members of control groups \{ group (II) and group (III) \}. Tuberculin skin tests were positive in 46(92\%) tuberculous patients of group (I), 7 of 20 (35\%) non-tuberculous patients and 3 of 15 (20\%) individuals of group III. Conclusion: Anti A60 IgG ELISA was the best serodiagnostic technique compared with Anti 60 IgM ELISA and
\end{abstract}


Mycodot test. The high diagnostic performance of Anti 60 IgG makes it a useful, Simple and rapid supporting tool to confirm the clinical judgment of tuberculosis when used as an adjunct to symptoms and signs together with other investigation tools.

\section{INTRODUCTION}

Tuberculosis (TB), as one of the mankind plagues and a major health concern shows resurgence in prevalence and intensity in both developing and well developed countries. One third of the world's population is infected with Mycobacterium tuberculosis. Three million people die every year of the disease with 9 million new cases per year ${ }^{(1)}$. TB has been a cause of significant morbidity and mortality for mankind throughout history ${ }^{(2)}$. Diagnosis of pulmonary tuberculosis depends mainly on the initial clinical suspicion and radiographic findings with subsequent microbiologic confirmation by direct smear microscopy and culture of sputum for acid fast bacilli (AFB). Some of the disadvantages of traditional diagnostic techniques are the lack of sensitivity in smear microscopy and the length of time in case of culture for AFB, sometimes growth takes several weeks ${ }^{(3,4)}$. Therefore, a number of alternative diagnostic tests that use molecular, chromatographic and immunological methods have been developed. Immunological methods use the specific humoral or cellular responses to investigate the presence of infection or disease. Numerous serological tests that use various antigens, such as secreted and heat shock proteins, lipopolysaccharides and peptide have been developed. These tests use various modification of enzyme- Linked immunosorbent assay (ELISA) or immunochromatographic methods to detect different antibody classes ${ }^{(5,6)}$. However, serological testing has been confounded by cross reactivity associated with bacillus calmette Guerin (BCG) vaccination or infection with mycobacteria other than tuberculosis (MOTT).

Previous serodiagnostic techniques have utilized either a mixture of $M$. tuberculosis antigens, such as purified extracted glycolipids, adsorbed mycobacterial sonicates, PPD or more distinct mycobacterial antigens $^{(5,7-11)}$. A study comparing three different antigen antibodies showed that A60 IgG (sensitivity and specificity, 80.77 and $88.4 \%$ ) was more antigenic and more effective in its determination than was $38 \mathrm{kDa} \operatorname{IgG}$ (sensitivity and specificity, 64.21 and $80.74 \%$ ) or KP90 IgA (sensitivity and specificity, 62.58 and $66.3 \%)^{(6)}$. The results of other serologic tests studies, including immunoglobulins to diacyltrehaloses, triacyltrehaloses, cord factor and sulpholipid I showed relatively low sensitivity and specificity for cases of tuberculosis infection ${ }^{(9)}$. The use of serological methods to diagnose tuberculosis has been studied since 1898 and A60 IgG, thermostable component of PPD, has been also used in the serodiagnosis of TB. Unfortunately, this molecule is not specific for mycobacteria because it is also present in Nocardia and cornybacterium species $^{(5,12-16)}$. In the 
present study, we aimed to evaluate IgG and IgM using A60 antigen by ELISA technique and Mycodot test using a combination of anti mycobacterial antigens (Lipoarabinomannan) for serodiagnosis of pulmonary tuberculosis in comparison with conventional bacteriological methods for laboratory diagnosis, namely microscopical examination of $\mathrm{ZN}$ stained sputum smear, culture and identification for acid fast bacilli (AFB).

\section{PATIENTS \& METHODS}

From September 2004 to September 2006, a prospective case control study was performed. The study included 3 groups: 50 adult patients with confirmed diagnosis of tuberculosis (acid fast smear, culture, tuberculin skin test, X-ray), 25 patients with chest diseases other than tuberculosis and 25 apparently healthy individuals. The members of group II\& group III participate as control groups.

Blood and sputum samples were collected from all subjects of group I and group II from Giza Chest Hospital, while those of group III were healthy volunteers. Sera were separated from blood samples and stored at $-20^{\circ} \mathrm{C}$ with $0.1 \%$ sodium azide. Sputum samples were submitted for direct microscopic examination for smears stained by $\mathrm{ZN}$, culture for AFB and identification of isolates. Serum samples were submitted for performing the serological tests, Mycodot test which utilizes lipoarabinomannan LAM antigen bound to plastic combs revealing pink spot when reacting with specific antibody present in serum. ELISA was performed to detect IgG and IgM using A60 antigen. Statistical analysis of results and cutoff values were established by using the receiver operating characteristic (ROC) curve technique.

\section{RESULTS}

\section{A) Mycodot test Results:}

The results of Mycodot test were positive in 33 cases out of 50 in the first group (confirmed tuberculous cases), while were positive in 6 cases out of 25 in the second group (nontuberculous pulmonary cases) and were positive in 3 cases out of 25 in the third group (apparently healthy individuals), the ROC curve has been shown in figure (1). From ROC curve of Mycodot test, the sensitivity and specificity were $66 \%$ and $82 \%$, while positive predictive value and negative predictive value were $78.57 \%$ and $70.69 \%$ respectively.

\section{B) IgG test results:}

The results of $\mathrm{IgG}$ test were positive in 42 cases out of 50 in the first group, 10 cases out of 25 in the second group and 8 cases out of 25 in the third group. From ROC curve of IgG test (figure 2), the sensitivity and specificity were $80 \%$ and $92 \%$, while positive predictive value and negative predictive value were $90.91 \%$ and $82.14 \%$ respectively.

\section{C) IgM test results:}

The results of $\operatorname{IgM}$ test were positive in 30 cases out of 50 in the first group, 3 cases out of 25 in the second group and 2 cases out of 25 in the third group. From ROC curve of IgM test (figure 3 ) the sensitivity and 
specificity were $82 \%$ and $74 \%$, while positive predictive value and negative predictive value were $75.93 \%$ and $80.43 \%$ respectively.

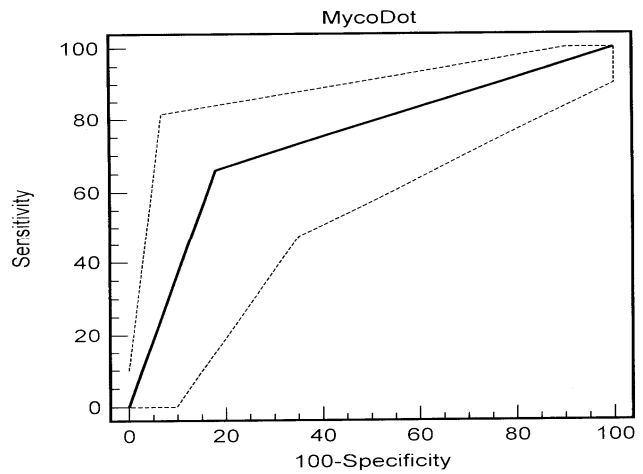

Fig. No. (1): ROC Curve of Mycodot test
- The comparison of the three ROC curves of the serological tests has been shown in Figure (4)

- The evaluation parameters of performance of the serological tests are summarized in (Table 1).

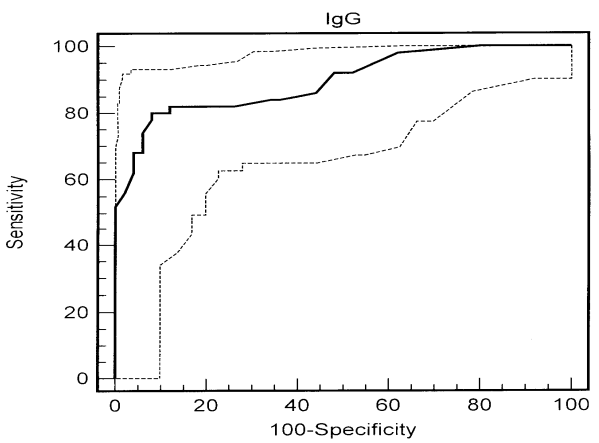

Fig. No. (2): ROC Curve of IgG test

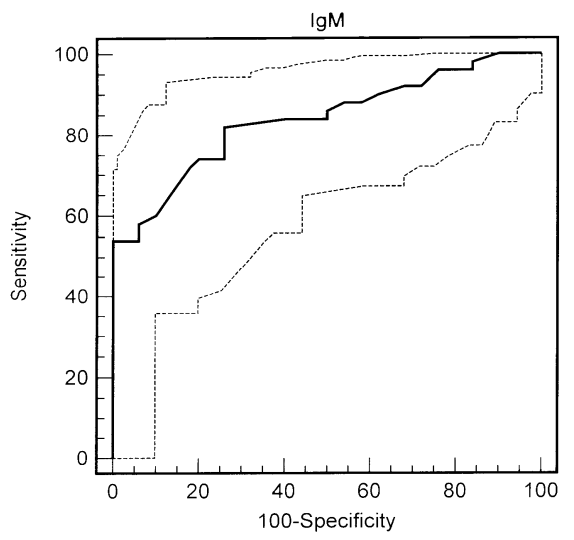

Fig. No. (3): ROC Curve of IgM test

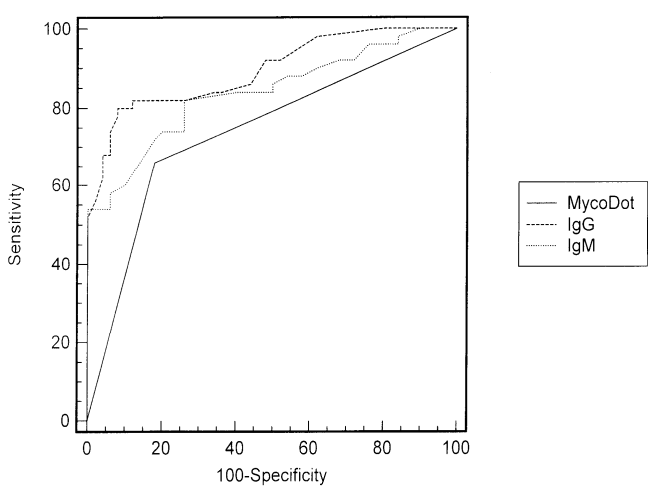

Fig. No. (4): Comparison of ROC curves for the three serological tests 
Table No. (1): Evaluation of Performance of Serological Tests

\begin{tabular}{l|ccc}
\hline \multirow{2}{*}{ Evaluation Parameter } & \multicolumn{3}{|c}{ Serological Tests } \\
\cline { 2 - 4 } Sensitivity & Mycodot & IgG & IgM \\
Positive Predicative Value & 66 & 80 & 82 \\
Specificity & 78.57 & 90.91 & 75.93 \\
Negative Predicative Value & 82 & 92 & 74 \\
\hline
\end{tabular}

\section{DISCUSSION}

A tentative diagnosis of TB can be made by observing acid-fast bacilli in smear of sputum, bronchoalveolar lavage, body fluids and gastric washing or urine, etc., while definite diagnosis is established only after the isolation and identification of tubercle bacilli from the patient ${ }^{(17)}$ and till now that method is considered to be the gold standard for diagnosing tuberculosis. However, invasive procedures are often required to obtain samples and the growth time of M. tuberculosis is unacceptably long. Other causes of granuloma formation may cause false positive results on

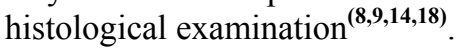

Despite the increasing development of techniques of rapid identification of mycobacteria by molecular genetic means, there is a hardly need for simple, sensitive and specific test for TB, which would improve or may replace the conventional methods ${ }^{(\mathbf{1 9 , 2 0})}$.

Methods based on Molecular biology are costly and complicated, so that they are not useful for the routine diagnosis in low-income

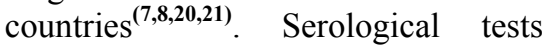
may be especially useful for a rapid diagnosis of $\mathrm{TB}$ in these countries, which may be over $90 \%$ of the global burden of TB cases $^{(\mathbf{2 0 , 2 1 )}}$. The aim of the present study was to evaluate the diagnostic usefulness of three serological tests, namely Mycodot test using LAM antigen, IgG\& IgM tests by ELISA using A60 antigen in pulmonary tuberculosis in adults.

Diagnostic accuracy of a test depends on the type of antigens used and on the population examined. For both adults and children, specificity of IgG assays based on recombinant antigens was very high (97-99\%). The specificity of assays based on native antigens were lower, which may be have been resulted from the cross reactivity of native antigens with environmental mycobacteria ${ }^{(9)}$. In most of the studies, it has been shown that IgG holds the great promise in diagnosing an active disease in both children as well as adults, when compared with IgM or Ig A classes. $\mathrm{IgG}$ is also found to be a useful antibody for monitoring the response of antituberculous treatment. IgG levels have been found to be increased in active TB. Increased IgM levels have also been reported in two studies, while several studies revealed no significant change. $\operatorname{IgM}$ is found to be the initial antibody produced. This feature suggests that the presence of IgM antibody to TB protein antigen might be characteristic of early stage 
of the disease, which may not hold diagnostic promise because the delay on the part of the patients in visiting qualified doctors ${ }^{(3,14-16 \& 18,22-25)}$.

In the present study, patients of group I with pulmonary tuberculosis showed high reactivity rate in the three serological tests compared with those of group II (non-tuberculous pulmonary patients) and those of group III (healthy volunteers). The sensitivity and specificity of $\mathrm{IgG}$ against A60 were $80 \%$ and $92 \%$ which agreed with other results, where they were found to be $(36,91 \%)$ and $(68,98 \%)$ respectively ${ }^{(26,27)}$.

These results are similar or mildly lower than those of other authors where the positivity rate ranges from $36 \%$ to $85 \%{ }^{(\mathbf{2 6 , 2 8})}$. This may be because of the population chosen or the criteria that the authors used for diagnosis. The result of $\mathrm{IgG}$ in the present study agreed with that of Alifano et al. ${ }^{(14)}$ which showed sensitivity and specificity of $\mathrm{IgG}$ $73.8 \%$ and $96.1 \%$ respectively and disagreed with that of Kochak et al. ${ }^{\text {(36) }}$ which showed sensitivity and specificity $54.3 \%$ and $84.2 \%$ and that of Turneer and vonNerom ${ }^{(29)}$ which showed positivity level of $29 \%$. The measurement of $\operatorname{IgM} \mathrm{A} 60$ in the present study differed with that of Yüce et al. ${ }^{(30)}$ which showed sensitivity and specificity of $43.8 \%$ and $97.04 \%$ respectively. The sensitivity value of $\operatorname{IgM}$ was previously reported to be between $24 \%$ and $60 \%{ }^{(26,27,31-33)}$. The results of Mycodot test may agreed with that of Abebe et al. ${ }^{(34)}$ that showed sensitivity and specificity of $89.3 \%$ and $100 \%$ and disagreed with that of ${ }^{(35)}$ which showed sensitivity and specificity of $18.5 \%$ and $100 \%$ and it was found that the concentration of antibodies against LAM antigen were elevated in relapse tuberculosis than that of newly acquired TB. In the present study, the detection of IgG by ELISA using A60 was the best method in diagnosing pulmonary tuberculosis as a sensitive and rapid serological tool of investigation compared with IgM A60 by ELISA and Mycodot test using LAM antigen where their sensitivities and specificities were $(80,92 \%),(82,74 \%)$ and $(66,82 \%)$ respectively.

So, it is recommended that, a development of serodiagnostic techniques for TB should be undertaken to define the target antigen(s) of Mycobacterium tuberculosis and the isotype(s) of antibody classes which is responsible for immune response against that target antigen(s).

\section{Acknowledgement:}

Many thanks, gratitude and appreciation to everyone participated in this work even with an encouragement word.

\section{REFERENCES}

1. World health organization (WHO report 2007): Global tuberculosis control, surveillance, planning, financing, Geneva, WHO, 277.

2. Reichman L.B. and Hershfield E.S. (1993): Tuberculosis. Marcel Dekker, Inc., New York Basel - Hong Kong.

3. Bhatia AS, kumar S, Harinath BC. (2003): Immunodiagnosis of tuberculosis: An update Indian J Clin. Biochem., 18:1-5. 
4. Julian E, Cama M., Martinez P. Lquin M (2001): An ELISA for five glycolipids from the cell wall of Mycobacterium tuberculosis: Twenty interferences in the assay. J. Immunol. Methods.,251: 21-30

5. Chan ED, Heifets $L$, Iseman MD (2000): Immunologic diagnosis of tuberculosis: a review Tuber. Lung Dis., 80 (3): 131-40.

6. Chiang IH, Suo J, Baik J, Lin TP, Luh KT, Yu CJ, et al., (1997): Serodiagnosis of tuberculosis. A study comparing three specific Mycobacterial antigens. Am. J. Respir. Crit. Care Med., 156 (3pt1): 906-11.

7. Pottumarthy S, wells VC, Morris AJ (2000): A comparison of seven tests for serological diagnosis of tuberculosis, J. Clin. Microbiol., 38(6) : 2227 - 31

8. Kalantri Y, Hemvani N, Bhatia GC, Chitinis DJ (2005): ELISA evaluation for $\operatorname{IgG}$ and $\operatorname{IgM}$ antibodies to A-60 tubercular protein antigen. Indian J. Med. Sci., 59 (8) : 337 - 46.

9. Wu HP, shieh WB, Hsien FK, Hua CC (2004): The significance of mycobacterium tuberculosis antibody, antigen A60 IgG in patients with abnormal chest radiography, Chang Gung Med. J., 27 (12) : 869 - 76.

10. Gennaro ML (2000): Immunological diagnostic methods of tuberculosis. Clin. Infect. Dis., 30 (sup 3) : 5243 - 6.

11. Wolinsky E. (1994): Conventional diagnostic methods for tuberculosis. Clin. Infect. Dis., 19 (3): 396 - 401.
12. Anuradha S, Kaur R, Singh NP, Baveja UK (2001): Serodiagnosis of extrapulmonary tuberculosis using A-60 antigen. J. Commun. Dis., 33 (1) : 12 -16.

13. Ng TT, Strong JI, wikins EG (1995): Serodiagnosis of pericardial tuberculosis, QJM., 88 (5) : $317-20$.

14. Alifano M, pascalis R, Sofia M, Faraone S, Del Pezzo M, Cevelli I (1998): Detection of IgG and Ig A against the mycobacterial antigen A60 in Patients with extrapulmonary tuberculosis, Thorax 53 (5) : $377-80$.

15. Bothamley G, Batra $H$, Ramesh V, Chandramui A. Ivanyi J (1992): Serodiagnostic value of 19 Kilodalton antigen of Mycobacterium tuberculosis in Indian patients. Eur. J. Clin. Microbiol. Infect. Dis.,11 (10) : 912 - 5

16. Hamasur B, Bruchfeld J, Haile M, Pawlowski A, Bjorvatn B, Kallenius G, et al. (2001): Rapid diagnosis of tuberculosis by detection of mycobacterial lipoarabinomannan in urine. J. Microbiol. Methods 45 (1) : 41 52.

17. Volk AW, Bryan M, Marie Louise H, Robert JK (1996): Essential of Medical Microbiology Laboratory diagnosis of tuber-culosis. Mycobacterium chapter; $31: 429$ - 39, fifth edn. copyright $(\mathrm{C}$ by Lippincott- Raven Publishers.

18. Ahmed $Z$, Pandey DK, Big M (2002): Role of anti -A60 IgG antibodies in diagnosis of tuberculous lymphadenitis, Ind. J Tub., 49: $101-4$. 
19. Raviglione MC, O'Brien RJ (1993): Tuberculosis In : Braunwald E, Hauser SL, Fauci AS, Longo DL, Kasper DL, Jameson JL, editor. Harrison's principles of internal medicine, 15th edn. New York : McGraw Hill; P. 1024 - 35

20. Demokow U, Ziolkowski J, Filewska M, Bialas-chromiec B, Zielonka T, MichalowskaMitczuk D, et al. (2004): Diagnostic Value of different serological tests for tuberculosis in Poland. J. physiol. Pharmacol., (55 suppl. 3) : 57 - 66

21. Ajei AA, Armah H, Duah OA, AdikuT, Hesse IF (2003): Evaluation of rapid serological chromatographic immunoassay for the diagnosis of pulmonary tuberculosis in Accra, Ghana, Jpn J. Infect. Dis., 56 (4) : $161-4$.

22. Jain VK, Bishnoi HS, Beniwal OP, Misra SN (1984): Immunoglobulin profile in pulmonary tuberculosis J. Postgrad. Med., 30 (2) : $80-4$.

23. Perkins MD, Conde MB, Martins M, Kritski AL (2003): Serologic diagnosis of tuberculosis using a simple Commercial multiantigens assay. Chest 123 (1) : $107-12$.

24. Zielonka TM, Demokow U, Filewska M, Bialas-chromiec B, Boros P, Zaleska J, et al., (2002): Usefulness of antibodies against A60 mycobacterial antigen in tuberculosis diagnosis. Pol. Merkur. Lekarski., 12 (72): 486-90.

25. Grubek-Jaworska H, Zwolska Z, Droszcz P, Rybus L, Dabrowski A, Droszcz W
(1997): Serum and bronchoalveolar IgG against A60 and $38 \mathrm{kDa}$ antigens in the diagnosis of tuberculosis. Int. J. Tuberc. Lung Dis., 1 (6) : 556 62.

26. Zou YL, Zhang JD, Chen MH, Shi GQ, Prignot J, Cocito C. (1994): Serological analysis of pulmonary and extrapulmonary tuberculosis with enzyme linked immunosorbent - assays for anti- A60 immunoglobulins. Clin. Infect. Dis., 19(6): 1084 - 91

27. Li LF, Lin MC, Chen NH, Hsich MJ, Lee CH, Tsao TC (1998): Serodiagnosis of tuberculosis by enzyme-linked immunosorbent assay for anti-60 and anti-A38. Chang Keng I Hsueh Tsa Chih., 21 (3) : 258 264

28. Baelden $M$, Vanderelst $B$, Dieng M, Prignot J, Cocito C (1990): Serological analysis of human tuberculosis by an ELISA with mycobacterial antigen A60. Scand. J. Infect. Dis., 22: 63 - 73.

29. Turneer $M$ and von Nerom $E$ (1993): Serological comparison of purified antigens 60 and 85A(P. 32) of Mycobacterium bovis $\mathrm{BCG}$, and purified protein derivative, in active pulmonary tuberculosis Eur. J. Epidemiol., 9 : 541 - 546

30. Yüce AM,Yücesoy S,Fence M,Sayan ES (2001): Serodiagnosis of tuberculosis by enzyme-immunoassay using A60 antigen. Clinical Microbiology and Infection 7 (7), $372-376$

31. Maes R (1991): Clinical usefulness of serological measurements obtained by 
antigen 60 in mycobacterial infections: development of a new concept. Klin. Wochenschr., 69 : $696-709$

32. Gupta S, KumariS, Bon waliker JN, Gupta SK (1995): Diagnostic utility of the estimation of mycobacterial antigen A60 specific immunoglobulins IgM, IgA and IgG in the sera of cases of adult human tuberculosis. Tub. Lung Dis., 76, $418-424$.

33. Simmoney N, Molina JM, Molinard M, Okseuhendler E, Lagrange H (1996): Comparison of A60 and the glycolipids antigens in an ELISA test for tuberculosis. Clin. Microbiol. Infect.., 2 (3) : $214-22$.
34. Abebe F, Holm-Hansen C, Wiker HG, Bjune G (2007): progress in serodiagnosis of mycobacterium infection. Scandinavian Journal of Immunology 66 (2-3): 176 - 191.

35. Julian E, Matos L, Ausina V, Luquin $M$ (1997): Detection of Lipoarabinomannan antibodies in patients with new acquired tuberculosis and patients with relapse tuberculosis. J. Clin. Microbiol ., 35 (3) : $535-7$.

36. Kochak HE, SevedAlinghi S, Zarghom O, Hekmat S,Jam S, Sabzvari D, Abdi $Z$ (2010): Evaluation of serological tests using A60 antigen for diagnosis of tuberculosis. Acta Med. Iran. Jan-Feb., 48 (1): 21 - 6.

\section{تقييم اختبار ات مصلية للتشخيص المناعي للارن الرئوي باستخدام}

\section{(A60 and Lipoarabinomannan antigens)}

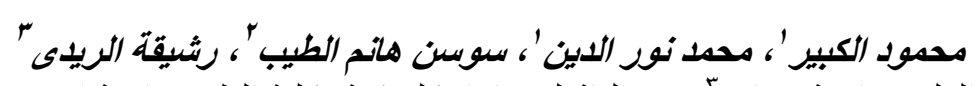

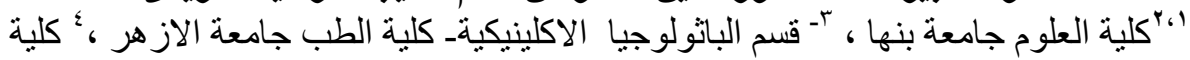
العلوم جامعة الةاهرة.

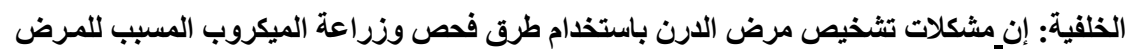

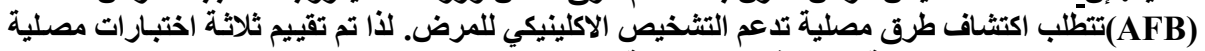

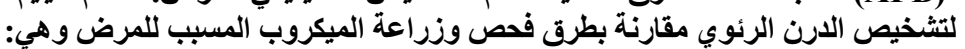

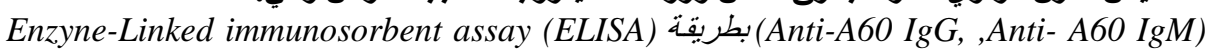

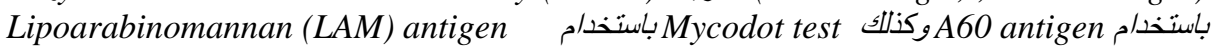

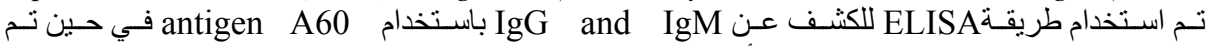

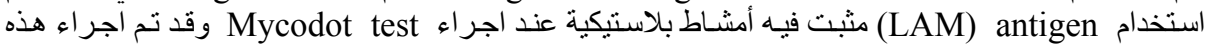

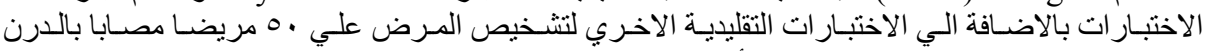

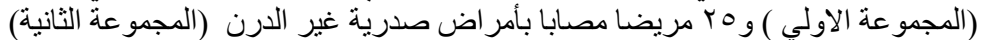

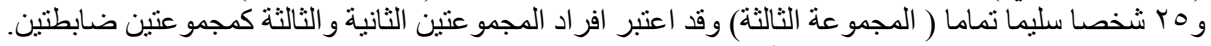

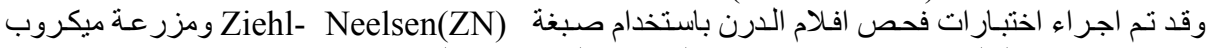

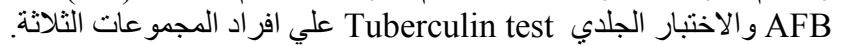

التتائج : 


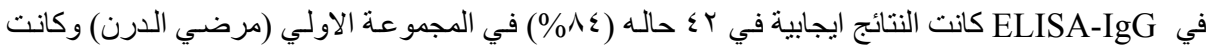

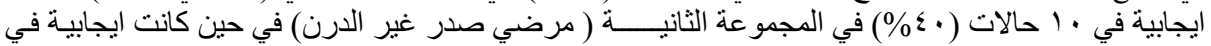

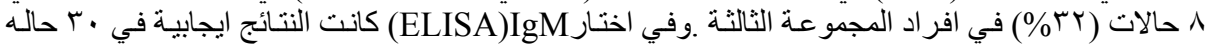

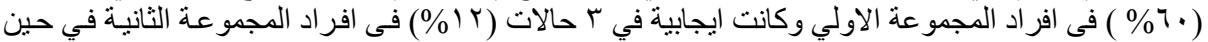

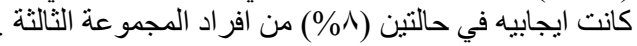

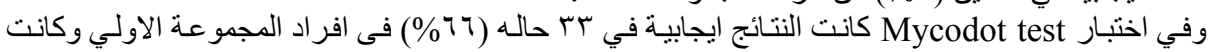

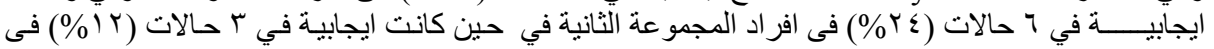

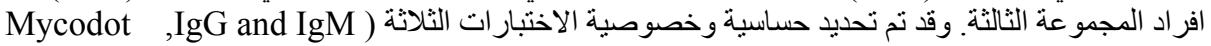

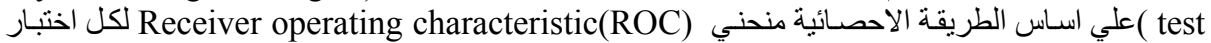

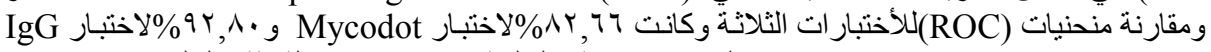

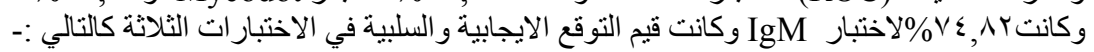

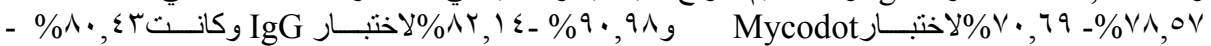

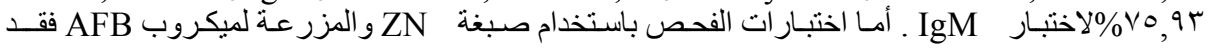

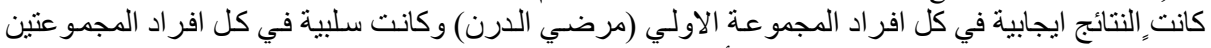

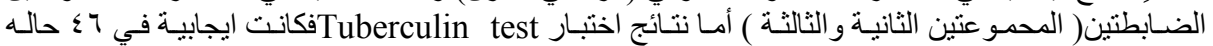

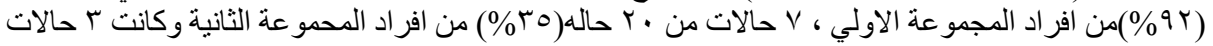

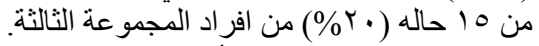

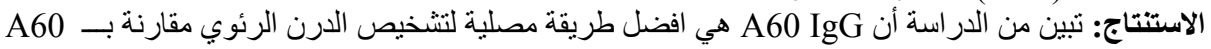

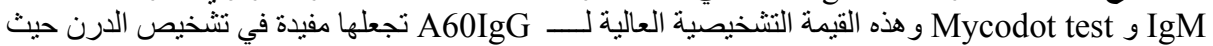
انها طريقة سريعة وقليلة التكلفة و عامل مدعم للتشخيص الاكلينيكي بالاضافة لطرق النشخيص الاخري. 